\title{
3D Nanotomography of Porous Polymer Composite using FIB/HIM and FIB/SEM
}

\author{
Doug Wei ${ }^{1}$, Stephan Kraemer ${ }^{2}$, Chunjie $\mathrm{Cao}^{3}$ and Chuong Huynh ${ }^{1}$ \\ 1. Ion Microscopy Innovation Center, Carl Zeiss Microscopy, LLC, Peabody MA, USA. \\ 2. Center for Nanoscale Systems, Harvard University, Cambridge MA, USA. \\ 3. Beijing Applications Laboratory, Carl Zeiss Microscopy, Inc, Beijing, China.
}

Recent development of helium ion microscopy (HIM) has enabled it as a two-column three-beam workstation with helium and neon ion beams generated from a GFIS column and gallium ion beam from a Ga-FIB column mounted on the microscope chamber at $54^{\circ}$ angle to the stage. This is a typical configuration of Orion NanoFab model in the HIM family. With this setup, the NanoFab system is capable of performing serial sectioning and imaging where sectioning is performed with the Ga ion beam and imaging is normally done using the He ion beam and, sometimes, Ne ion beam.

As reported in literature [1-2], imaging with He ion beams has shown some advantages compared to SEM imaging, including high spatial resolution $(<0.5 \mathrm{~nm})$, high depth of field, high surface sensitivity and charge neutralization with low energy electron flooding, etc. Here we report a study of 3D nanotomography of a porous polymer composite using FIB/HIM through serial sectioning and imaging. This is the first time a FIB/HIM (Orion NanoFab) is used for 3D nanotomography of such a material system. 3D nanotomography studies of soft/bio materials using FIB/SEM technology has been well understood and high resolution volume imaging down to $3 \mathrm{~nm}$ voxel size has been reported [3]. We have included FIB/SEM (Helios Nanolab 660) results of the same sample here as a bench mark for comparison.

The material system studied in this report is a porous EVA polymer matrix in which pores were formed by leaching away the water soluble component. While a $30 \mathrm{keV} 1.5 \mathrm{nA}$ Ga ion beam was used in both systems for milling (no observable ion beam damage), a 30keV $1 \mathrm{pA}$ He ion beam was employed for imaging in the FIB/HIM and a 2keV 200pA electron beam in the FIB/SEM (low voltage for high surface sensitivity and low beam currents for minimizing radiation damage). Images were recorded in immersion mode to achieve high detection yield and both secondary and backscattered electrons have been collected.

Representative morphologies of a cross-section of the polymer matrix are shown in Figure 1: a) an HIM image, and c) an SEM image (SE mode). The most ideal gray scale intensity distribution with respect to segmentation of a 3D datacube into bulk and pore regions is bimodal. It can be seen that the HIM images come most closely to such an ideal intensity distribution: very few secondary electrons escape the pores, which is probably due to local positive charging within the pores. SEM images, on the other hand, show significantly more structural details inside the pores with often higher intensities emitted from the pores than the surface. One potential factor might be redeposition of sputtered materials (containing Ga ions). Interestingly there was no significant difference when in-lens detector was used in image formation as compared to backscattered electron mode.

Image stacks of about 800-1000 slices with $20 \mathrm{~nm}$ voxel size were acquired on both systems. ORS software was employed for 3D image processing, including image alignment, 3D reconstruction, segmentation and rendering. Pore spaces in the HIM images can easily be selected using gray-scale 
based automated segmentation (Fig. 1b). However, segmenting pore spaces out of the solid continuous phase in the polymer matrix in the SEM images appears to be much less straight-forward, where besides the polished surface also regions within the pores are selected (Fig. 1d). It is noted that the systematically brighter edges separate most of those regions from the bulk continuous region, allowing at least partially to remove them a posteriori via morphological operations. The advantages of SEM over HIM in two dimensional imaging become a burden in 3D tomographical study. In this case, the computed porosity from 3D volume image reconstructed from the HIM image stack is about $44.6 \%$ while that from the SEM image stack is only about $9.5 \%$ when gray-scale based automated segmentation protocol is employed. If the SEM images were segmented manually based on human judgement, the computed porosity becomes $47.8 \%$, very close to the actual porosity value of $50 \%$.

$3 \mathrm{D}$ rendering of the FIB/HIM image stack was performed using the ORS software and the pore space within the imaged volume is presented in Figure 2.
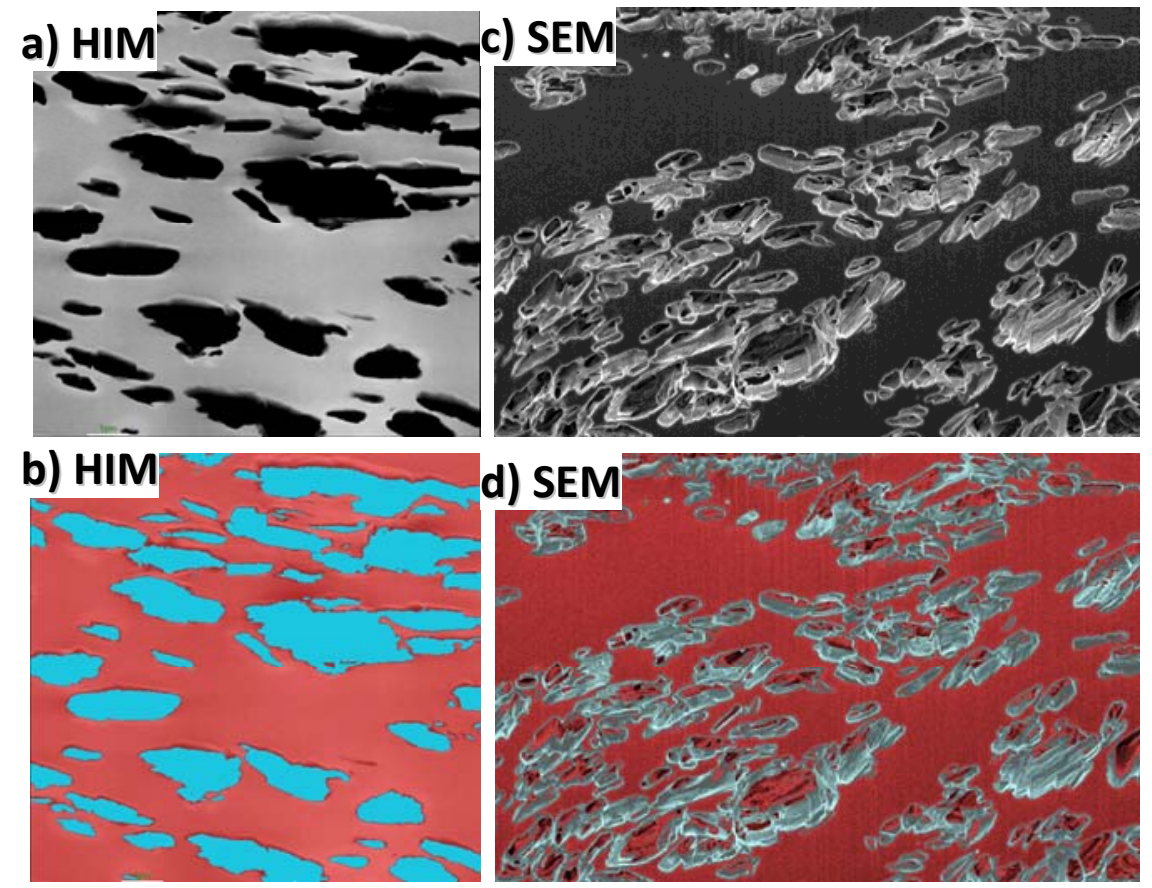

Figure 1. Representative slices from the HIM and SEM image stacks, and pore space segmentations based on gray-scale.

In conclusion, this preliminary investigation of 3D nano-tomography of the porous polymer composite using the Orion NanoFab FIB/HIM system and the Helios Nanolab 660 FIB/SEM system helped us understand the benefits of both technologies in imaging such a material system. While we have seen rich information in SEM images, especially structural details inside the pores, HIM imaging really demonstrated advantages in 3D imaging, i.e., making the downstream automated image processing much easier, resulting more reliable analysis results.

\section{References:}

[1] M Joens, C Huynh and J. Fitzpatrick, et al, Scientific Reports 3 (2013) Article number: 3514.

[2] R Hill, J Notte and L Scipioni, Scanning Helium Ion Microscopy (Chapter 2 in Advances in Imaging and Electron Physics) 2012.

[3] D Wei, K Czymmek, et al, BioTechniques, Vol. 53,

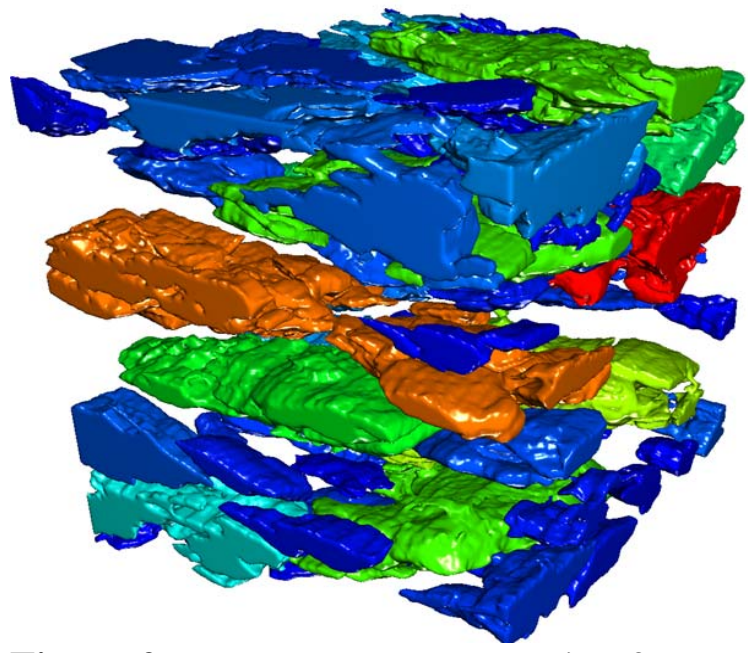

Figure 2. Pore space representation from the $3 \mathrm{D}$ rendering of the FIB/HIM image stack.

No. 1 (2012) pp. 41-48. 